\title{
PERFIL SOCIOECONÔMICO E NUTRICIONAL DE PACIENTES COM DOENÇA INFLAMATÓRIA INTESTINAL INTERNADOS EM UM HOSPITAL UNIVERSITÁRIO
}

\author{
Flávia Nunes SALVIANO, Maria Goretti Pessoa de Araújo BURGOS e Eduila Couto SANTOS
}

\begin{abstract}
RESUMO - Racional - Doenças inflamatórias intestinais incluem distúrbios inflamatórios crônicos e recidivantes, representados pela retocolite ulcerativa e doença de Crohn, comumente associadas à desnutrição. Objetivo - Caracterizar o perfil nutricional e socioeconômico dos pacientes internados no Hospital das Clínicas de Pernambuco, Recife, PE. Métodos - Estudo do tipo transversal realizado na clínica de gastroenterologia, previamente aprovado pela comissão de ética para estudos em humanos. Os métodos incluíram dados da história clínica, condições socioeconômicas e avaliação nutricional. A análise estatística (teste $t$ de Student para variáveis iguais e desiguais) foi efetuada. Resultados - A amostra de 24 pacientes de ambos os sexos, idade média de 43,83 $\pm 16,13$ anos, a maioria casados, procedentes de Recife, com baixa renda, residindo com seus familiares em casa própria. Verificou-se maior prevalência de retocolite ulcerativa $(62,5 \%)$, com tempo de diagnóstico superior a 5 anos, sintomas de dor abdominal, diarréia muco-sangüinolenta com 6-9 evacuações/dia, sendo o cólon distal a porção mais acometida. A osteoporose esteve presente em $26,7 \%$ dos casos. O estado nutricional avaliado através do índice de massa corpórea e percentual de perda de peso foi $41,7 \%$ e 70,8\%, respectivamente, classificados como desnutridos, associados ou não à alta prevalência de anemia, hipoalbuminemia e hipocalcemia. Correlação entre sexos evidenciou nos homens maiores valores na prega tricipital e circunferência do braço. Conclusões - Apesar das limitações do estudo, os dados sugerem informações relevantes tanto da ocorrência destas doenças na região nordeste do país, quanto sua associação freqüente com importantes deficiências nutricionais.
\end{abstract}

DESCRITORES - Enteropatias inflamatórias. Doença de Crohn. Proctocolite. Avaliação nutricional. Fatores socioeconômicos.

\section{INTRODUÇÃO}

As doenças inflamatórias intestinais (DII), classicamente conhecidas como retocolite ulcerativa inespecífica (RCUI) e doença de Crohn (DC) são processos inflamatórios crônicos que cursam de maneira imprevisível com períodos de atividade e remissão variáveis; apesar de sintomatologia clínica semelhantes, são consideradas doenças distintas ${ }^{(26)}$.

Descritas em todo o mundo, há tendência do crescimento de suas incidências ${ }^{(15,22)}$ inclusive no Brasil ${ }^{(26,48)}$, embora sejam escassos os estudos sobre a ocorrência da DC e da RCUI nos países em desenvolvimento ${ }^{(30)}$, principalmente no nordeste brasileiro. Predominam em raça branca (nórdicos europeus) $^{(18,52)}$, apresentando distribuição semelhante em ambos os sexos, com a DC atingindo mais a população feminina, com idade mais freqüente em adultos jovens, na faixa etária entre 20 a 40 anos e um segundo pico a partir dos $55 \operatorname{anos}^{(40)}$. Prevalece, ainda, em áreas urbanas em relação às rurais e em classes econômicas mais altas ${ }^{(10)}$, em fumantes e parentes de primeiro grau de indivíduos acometidos ${ }^{(18)}$.

A compreensão da etiologia e patogênese das DII melhorou extensivamente nos últimos anos, provavelmente por maior entendimento do sistema imunológico do intestino e particularmente sua reação inflamatória decorrente na flora intestinal normal ${ }^{(5,46)}$. Acredita-se que a doença tenha etiopatogenia multifatorial, com participações de fatores genéticos, ambientais, microflora intestinal e reposta imune. No que se refere aos fatores genéticos e ambientais, são mal definidos e não são ainda sensíveis às manipulações terapêuticas por razões técnicas e éticas ${ }^{(23)}$, apesar de descritas maiores incidências em gêmeos monozigóticos e grupos éticos específicos ${ }^{(18)}$. Com isso a microflora intestinal e a reposta imune passam a ser as duas categorias selecionadas para os tratamentos atuais ${ }^{(33,44,45)}$. 
Em relação à microbiota, sabe-se que pacientes com DII apresentam alterações qualitativa e quantitativa, mas nenhuma bactéria, isoladamente, pode ser responsável por desencadear o processo inflamatório ${ }^{(23)}$. Além desses fatores, existe uma alteração na permeabilidade da mucosa intestinal e resposta imunológica anormal, que ativa uma cascata imunoinflamatória, resultando em lesão continuada da mucosa intestinal ${ }^{(8)}$. A amplificação da reposta inflamatória é mais importante que o evento inicial como causa da destruição tecidual e das alterações histológicas e funcionais características das $\mathrm{DII}^{(18)}$.

As DII são comumente associadas à desnutrição proteicoenergética, como também à carência de micronutrientes tanto em pacientes hospitalizados como em ambulatório ${ }^{(16,17,27,31,49)}$, causadas por redução da ingestão alimentar, má absorção, aumento das perdas gastrointestinais e necessidades nutricionais aumentadas ${ }^{(29,42,43)}$. Essas alterações têm impacto em todas as idades, sofrendo a influência da fase de atividade, extensão e localização da doença ${ }^{(18,31,42)}$. A desnutrição, por sua vez, agrava o prognóstico tanto do paciente em tratamento clínico, quanto naqueles submetidos a cirurgias, além de deteriorar a competência imune ${ }^{(43)}$.

Segundo FISHER ${ }^{(24)}$, as deficiências nutricionais mais freqüentes são: perda de peso, hipoalbuminemia, balanço nitrogenado negativo, anemia e deficiência de vitaminas (Folato, B12, D) e minerais (Fe, Ca, Zn e Mg). De acordo com WAITZBERG e SILVA ${ }^{(53)}$, a avaliação nutricional permite o reconhecimento precoce da desnutrição e a terapêutica necessária para a sua correção. Além disso, um suporte nutricional adequado deve ser instituído com o objetivo de reduzir as indicações cirúrgicas e as complicações operatórias, mantendo e/ou recuperando as condições nutricionais ${ }^{(14,18)}$.

Considerando as características demográficas, clínicas e evolutivas dos pacientes com DII, este estudo ganha relevância devido à escassez de pesquisas regionais realizadas com esse objetivo. Portanto, pretende-se caracterizar esse grupo em um hospital do nordeste brasileiro, para acompanhamento multiprofissional específico e conseqüentemente, conduta dietoterápica mais adequada, proporcionando redução do tempo de hospitalização além de melhor qualidade de vida.

\section{MÉTODOS}

Estudo transversal, realizado na clínica de Gastroenterologia do Hospital das Clínicas de Pernambuco (HC/UFPE), Recife, PE, durante o período de julho a dezembro de 2005. O protocolo foi previamente aprovado pela Comissão de Ética para estudos em humanos do Centro de Ciências da Saúde da UFPE, de acordo com a Resolução no 196/96 do Conselho Nacional de Saúde, sob o número do registro 198/2005. Os critérios de inclusão foram: pacientes com diagnóstico de RCUI e DC em qualquer período de atividade da moléstia, de ambos os sexos, na faixa etária entre 20-80 anos. Os pacientes sem diagnóstico clínico definido através de exames radiológicos e histopatológicos, faixa etária abaixo de 20 anos e acima de 80 anos e aqueles tratados em ambulatório, foram excluídos da pesquisa.

A caracterização da população incluiu dados de identificação, história clínica e condições socioeconômicas (segundo a
Pesquisa Nacional de Orçamento Familiar 2002/2003) ${ }^{(9)}$, a partir da entrevista com o paciente (no momento da admissão na enfermaria), com informações contidas no prontuário e ficha de acompanhamento individual.

Em relação à faixa etária, foram divididos em três subgrupos: 20-40 anos, 41-59 anos, acima de 60 anos, classificados como adultos jovens, adultos e idosos, respectivamente. Quanto ao grau de instrução, foram classificados os níveis de escolaridade em analfabeto, $1^{\circ}, 2^{\circ}$ e $3^{\circ}$ grau completo. A renda familiar foi calculada a partir da soma das rendas mensais de todos residentes no domicílio, considerando-se o número de pessoas que residiam na mesma casa, independentemente de parentesco; adotou-se o ponto de corte em três níveis: $0-2,2-5,>5$ salários mínimos, considerando 5 salários mínimos como necessário para uma família de quatro membros.

Em relação às características clínicas, o tempo de diagnóstico foi descrito em anos e definido como o intervalo entre a confirmação diagnóstica (critérios radiológicos e histopatológicos) e a data do internamento atual, identificando-se os principais sinais e sintomas no momento do internamento hospitalar. O registro do número de evacuações e a gravidade da manifestação clínica foram comparados com a presença de perda de peso. Quanto à localização da doença, foram identificadas as principais porções gastrointestinais acometidas, através de laudos clínicos e histopatológicos. As manifestações extra-intestinais foram registradas, indicando a intensidade com que se manifestam as DII.

$\mathrm{Na}$ antropometria, foram utilizados métodos de aferição de altura e peso e calculado o índice de massa corporal (IMC) de acordo com classificação proposta pela Organização Mundial de Saúde ${ }^{(32)}$; prega cutânea do tríceps (PCT), circunferência do braço (CB) e circunferência muscular do braço (CMB), pelos critérios de BLACKBURN e THORNTON ${ }^{(7)}$. Além desses, foi avaliado o percentual de perda ponderal (\%PP) do indivíduo após a doença, calculado entre o peso habitual e o atual dividido pelo peso habitual, durante os últimos 3 meses, com classificação proposta por BLACKBURN et al. ${ }^{(6)}$, modificada por CARVALHO(19).

Os pacientes foram pesados no momento da admissão e semanalmente durante todo o internamento, utilizando-se balança de marca Filizola, com capacidade $150 \mathrm{~kg}$ e altura medida através de um antropômetro fixo à balança. As circunferências e a PCT foram mensuradas com plicômetro da marca Cescorf e fita métrica de fibra de vidro não-distensível, em centímetros, com precisão de milímetros, através das técnicas de medição proposta por LOHMAN et al. ${ }^{(37)}$. As medidas antropométricas descritas foram realizadas exclusivamente pelo próprio pesquisador, a fim de minimizar erros.

Em relação aos parâmetros bioquímicos, foram utilizadas dosagens séricas de albumina, hematócrito, hemoglobina, glicose em jejum, uréia, creatinina, sódio, potássio e cálcio, por serem as únicas de rotina do serviço e dosados no laboratório do HC/UFPE.

As comparações estatísticas foram realizadas pelo teste $t$ de Student para variâncias iguais e desiguais. A margem de erro (ou nível de significância) utilizado na decisão dos testes estatísticos foi de $5,0 \%{ }^{(2,54)}$. 


\section{RESULTADOS}

A amostra foi constituída de 24 pacientes, com maior predomínio de homens $(58,3 \%)$ e idade média de 43,83 \pm 16,13 anos. Com relação às características socioeconômicas, a maior parte da amostra tinha ocupação doméstica, seguida de ambulantes e estudantes, em sua maioria casados, procedentes de Recife, com baixa renda, residindo com seus familiares em casa própria. Quanto ao nível de escolaridade, foi observado que quase $50 \%$ tinha cursado o $2^{\circ}$ grau (Tabela 1 ).

Analisando as características clínicas da amostra, o percentual de RCUI foi superior aos de DC (62,5\% e 37,5\%,

TABELA 1. Condições socioeconômicas dos pacientes com DII

\begin{tabular}{|c|c|c|}
\hline Variável & $\mathrm{n}$ & $\%$ \\
\hline \multicolumn{3}{|l|}{ Profissão } \\
\hline Do lar & 6 & 25,0 \\
\hline Ambulante & 4 & 16,7 \\
\hline Estudante & 4 & 16,7 \\
\hline Comerciante & 3 & 12,5 \\
\hline Aposentado & 2 & 8,3 \\
\hline Eletricista & 2 & 8,3 \\
\hline Agricultora & 1 & 4,2 \\
\hline Motorista & 1 & 4,2 \\
\hline Marceneiro & 1 & 4,2 \\
\hline Total & 24 & 100,0 \\
\hline \multicolumn{3}{|l|}{ Estado civil } \\
\hline Casado & 16 & 66,7 \\
\hline Solteiro & 8 & 33,3 \\
\hline Total & 24 & 100,0 \\
\hline \multicolumn{3}{|l|}{ Procedência } \\
\hline Recife & 14 & 58,3 \\
\hline Região Metropolitana & 7 & 29,2 \\
\hline Interior & 3 & 12,5 \\
\hline Total & 24 & 100,0 \\
\hline \multicolumn{3}{|c|}{ Renda familiar (em salário mínimo) } \\
\hline De 0 a 2 & 12 & 50,0 \\
\hline De 2 a 5 & 10 & 41,7 \\
\hline De $>5$ & 2 & 8,3 \\
\hline Total & 24 & 100,0 \\
\hline \multicolumn{3}{|c|}{$\mathbf{N}^{\circ}$ de pessoas que reside na família } \\
\hline De 3 a 4 & 10 & 41,7 \\
\hline De 5 a 6 & 10 & 41,7 \\
\hline De 7 a 8 & 4 & 16,7 \\
\hline Total & 24 & 100,0 \\
\hline \multicolumn{3}{|l|}{ Condição do imóvel } \\
\hline Próprio & 15 & 62,5 \\
\hline Alugado & 9 & 37,5 \\
\hline Total & 24 & 100,0 \\
\hline \multicolumn{3}{|l|}{ Escolaridade } \\
\hline $2^{\circ}$ grau completo & 10 & 41,7 \\
\hline $1^{\circ}$ grau completo & 8 & 3,3 \\
\hline Analfabeta & 6 & 25,0 \\
\hline Total & 24 & 100,0 \\
\hline
\end{tabular}

respectivamente), com tempo de diagnóstico superior a 5 anos $(56,5 \%)$, apresentando dor abdominal e diarréia mucosanguinolenta como principais causas de internamento $(41,7 \%)$, com seis a nove evacuações por dia. A porção intestinal mais acometida foi o cólon distal (33,3\%), seguido por sigmóide e reto $(26,7 \%)$. Em relação às manifestações intra e extra-intestinais, $26,7 \%$ dos pacientes apresentaram osteoporose, seguido pela ocorrência de colostomia (13,3\%), ileostomia $(13,3 \%)$ e fístulas $(13,3 \%)$, sendo marcante o grau de intensidade.

Os resultados do perfil bioquímico revelaram alto percentual de hipoalbuminemia, prevalência elevada de anemia, observadas através dos níveis de hematócrito e hemoglobina. Por outro lado, a maioria dos pacientes apresentava a glicose de jejum, uréia, creatinina, sódio e potássio dentro dos padrões de normalidade. No que se refere ao cálcio sérico, constatou-se níveis reduzidos, demonstrando grande vulnerabilidade desses indivíduos em relação ao metabolismo ósseo.

De acordo com a adoção dos critérios da OMS de $1997^{(32)}$ para o IMC, no momento da admissão, 41,7\% apresentaramse como desnutridos. Enquanto o indicador de \% PP revelou percentual mais elevado de desnutrição, alcançando 70,8\% da amostra (Tabela 2). Destes, 64,7\% apresentavam perda de peso maior que $10 \%$ em menos de 6 meses. Na subdivisão da amostra por grau da desnutrição, constatou-se percentual elevado de desnutridos graves usando o indicador \% $\%$, como mostrado na Figura 1.

TABELA 2. Distribuição da amostra segundo o estado nutricional

\begin{tabular}{lcc}
\hline Variável & $\mathbf{n}$ & $\%$ \\
\hline Classificação do estado nutricional segundo o IMC & & \\
$\quad$ Eutrófico & 14 & 58,3 \\
$\quad$ Desnutrido & 10 & 41,7 \\
Total & 24 & 100,0 \\
\hline Classificação do estado nutricional segundo a perda de peso & & \\
$\quad$ Desnutrido & 17 & 70,8 \\
$\quad$ Eutrófico & 7 & 29,2 \\
Total & 24 & 100,0 \\
\hline
\end{tabular}

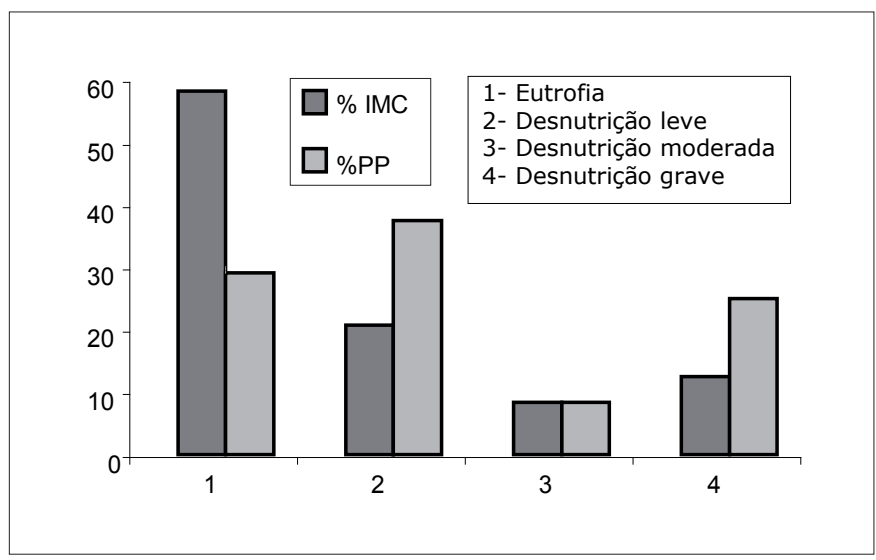

FIGURA 1. Comparação do IMC com o \%PP por grau de desnutrição 
No que se refere ao sexo e estado nutricional (Tabela 3), observou-se maior percentual de desnutrição no sexo feminino, segundo o IMC, enquanto o indicador de $\% \mathrm{PP}$ evidenciou maior número no sexo masculino. Quando se subdividiu a amostra por grau de desnutrição, as mulheres foram mais afetadas pela desnutrição grave. Na associação com as variáveis: $\mathrm{PCT}$, CMB e $\mathrm{CB}$, observou-se menores valores de $\mathrm{PCT}$ e $\mathrm{CB}$ em mulheres, estatisticamente significante (Tabela 4).

TABELA 3. Estado nutricional segundo o sexo nos pacientes com DII

\begin{tabular}{lcccc}
\hline \multirow{2}{*}{ Variáveis } & \multicolumn{4}{c}{ Sexo } \\
\cline { 2 - 5 } & \multicolumn{2}{c}{ Masculino } & \multicolumn{2}{c}{ Feminino } \\
\cline { 2 - 5 } & $\mathrm{n}$ & $\%$ & $\mathrm{n}$ & $\%$ \\
\hline Classificação segundo o IMC & & & & \\
$\quad$ Eutrófico & 9 & 64,3 & 5 & 50 \\
$\quad$ Desnutridos & 5 & 35,7 & 5 & 50 \\
Total & 14 & 100 & 10 & 100 \\
\hline Desnutrição leve & 3 & 21,5 & 2 & 20 \\
Desnutrição moderada & 1 & 7,1 & 1 & 10 \\
Desnutrição grave & 1 & 7,1 & 2 & 20 \\
\hline Classificação segundo o \% PP & & & & \\
$\quad$ Eutrófico & 3 & 21,5 & 4 & 40 \\
$\quad$ Desnutridos & 11 & 78,5 & 6 & 60 \\
Total & 14 & 100 & 10 & 100 \\
\hline Desnutrição leve & 8 & 57,1 & 1 & 10 \\
Desnutrição moderada & 1 & 7,1 & 1 & 10 \\
Desnutrição grave & 2 & 14,3 & 4 & 40 \\
\hline
\end{tabular}

Não houve correlação entre o estado nutricional e parâmetros bioquímicos, com exceção dos eletrólitos, sódio e potássio. A hipoalbuminemia esteve presente em $80 \%$ dos pacientes desnutridos, enquanto que a anemia não se mostrou dependente do estado nutricional avaliado pela antropometria (Tabela 5).

Os níveis de glicose foram semelhantes em ambos os grupos. Por outro lado, comportamento diferente foi detectado nos níveis de uréia e creatinina, em que os desnutridos apresentaram tendência elevada em relação aos eutróficos. Quanto ao cálcio, os valores encontravam-se normais apenas nos eutróficos de ambos os sexos (Tabela 5).

Como podem ser observados na Tabela 6 , sinais e sintomas, como diarréia, vômitos e dor abdominal, foram identificados com maior freqüência em pacientes desnutridos, assim como as complicações extra e intra-intestinais $(66,7 \%)$, ressaltando a importância da avaliação nutricional precoce.

\section{DISCUSSÃO}

A determinação das características socioeconômicas, clínicas e nutricionais dos pacientes com DII em um hospital universitário do nordeste brasileiro ganha importância devido à deficiência de estudos regionais. Assim sendo, não se dispõe de informações precisas sobre a ocorrência dessas enfermidades e tampouco sobre as dimensões da população de referência.

Os dados deste estudo revelam predomínio da amostra do sexo masculino, com faixa etária de 40 anos, diferindo dos resultados de SOUZA et al. ${ }^{(47)} \mathrm{e}$ VIEIRA et al. ${ }^{(51)}$, que observaram predomínio do

TABELA 4. Associação do estado nutricional segundo o sexo nos pacientes com DII

\begin{tabular}{|c|c|c|c|c|c|}
\hline \multirow[b]{2}{*}{ Variáveis } & \multirow[b]{2}{*}{ Estatísticas } & \multicolumn{2}{|c|}{ Sexo } & \multirow[b]{2}{*}{ Total } & \multirow[b]{2}{*}{ Valor $P$} \\
\hline & & Masculino & Feminino & & \\
\hline \multirow[t]{3}{*}{ Peso (kg) } & Média & 53,83 & 46,94 & 50,96 & $P^{(1)}=0,1156$ \\
\hline & Desvio padrão & 10,28 & 9,98 & 10,52 & \\
\hline & $\mathrm{CV}$ & 19,10 & 21,25 & 20,65 & \\
\hline \multirow[t]{3}{*}{ Altura (m) } & Média & 1,67 & 1,55 & 1,62 & $P^{(1)}=0,0026^{*}$ \\
\hline & Desvio padrão & 0,09 & 0,08 & 0,10 & \\
\hline & $\mathrm{CV}$ & 5,26 & 5,34 & 6,39 & \\
\hline \multirow[t]{3}{*}{$\operatorname{IMC}\left(\mathrm{kg} / \mathrm{m}^{2}\right)$} & Média & 19,50 & 19,49 & 19,50 & $P^{(1)}=0,9900$ \\
\hline & Desvio padrão & 3,25 & 3,38 & 3,23 & \\
\hline & $\mathrm{CV}$ & 16,65 & 17,37 & 16,57 & \\
\hline \multirow[t]{3}{*}{$\%$ de perda de peso } & Média & 12,41 & 16,49 & 14,11 & $P^{(1)}=0,4329$ \\
\hline & Desvio padrão & 10,84 & 14,23 & 12,24 & \\
\hline & $\mathrm{CV}$ & 87,31 & 86,30 & 86,75 & \\
\hline \multirow[t]{3}{*}{ Prega cutânea tricipital (mm) } & Média & 9,27 & 7,51 & 8,54 & $P^{(2)}=0,0288^{*}$ \\
\hline & Desvio padrão & 2,51 & 1,00 & 2,18 & \\
\hline & $\mathrm{CV}$ & 27,06 & 13,37 & 25,50 & \\
\hline \multirow[t]{3}{*}{ Circunferência do braço $(\mathrm{cm})$} & Média & 21,58 & 17,11 & 19,72 & $P^{(2)}=0,0483^{*}$ \\
\hline & Desvio padrão & 3,16 & 5,89 & 4,93 & \\
\hline & $\mathrm{CV}$ & 14,62 & 34,44 & 24,99 & \\
\hline Circunferência muscular & Média & 18,82 & 17,79 & 18,39 & $P^{(1)}=0,4296$ \\
\hline \multirow[t]{2}{*}{ do braço $(\mathrm{cm})$} & Desvio padrão & 3,12 & 3,06 & 3,07 & \\
\hline & $\mathrm{CV}$ & 16,56 & 17,19 & 16,69 & \\
\hline
\end{tabular}

* $=$ diferença significante ao nivel de $5,0 \%$

(1) = através do teste + de Student com variâncias iguais

(2) = através do teste + de Student com variâncias desiguais

$\mathrm{CV}=$ coeficiente de variação 
TABELA 5. Variáveis bioquímicas e estado nutricional dos pacientes com DII

\begin{tabular}{|c|c|c|c|c|c|}
\hline \multirow[b]{2}{*}{ Variáveis } & \multirow[b]{2}{*}{ Estatísticas } & \multicolumn{2}{|c|}{ Estado nutricional } & \multirow[b]{2}{*}{ Total } & \multirow[b]{2}{*}{ Valor $P$} \\
\hline & & Desnutrido & Normal & & \\
\hline \multirow[t]{3}{*}{ Albumina $(g / d L)$} & Média & 2,83 & 2,83 & 2,83 & $P^{(1)}=0,9889$ \\
\hline & Desvio padrão & 1,04 & 0,95 & 0,98 & \\
\hline & $\mathrm{CV}$ & 36,57 & 33,50 & 34,72 & \\
\hline \multirow[t]{3}{*}{$\mathrm{HT}(\%)$} & Média & 29,82 & 27,32 & 29,10 & $P^{(1)}=0,4536$ \\
\hline & Desvio padrão & 7,04 & 5,98 & 6,70 & \\
\hline & $\mathrm{CV}$ & 23,59 & 21,89 & 23,03 & \\
\hline \multirow[t]{3}{*}{$\mathrm{HB}(\mathrm{g} / \mathrm{dL})$} & Média & 9,90 & 9,02 & 9,65 & $P^{(1)}=0,4857$ \\
\hline & Desvio padrão & 2,77 & 1,91 & 2,54 & \\
\hline & $\mathrm{CV}$ & 27,98 & 21,20 & 26,33 & \\
\hline \multirow[t]{3}{*}{ Glicose jejum (mg/dL) } & Média & 97,70 & 85,50 & 93,63 & $P^{(1)}=0,5515$ \\
\hline & Desvio padrão & 36,60 & 18,91 & 31,41 & \\
\hline & $\mathrm{CV}$ & 37,47 & 22,12 & 33,54 & \\
\hline \multirow[t]{3}{*}{ Uréia $(\mathrm{mg} / \mathrm{dL})$} & Média & 34,21 & 19,32 & 29,51 & $P^{(1)}=0,0516$ \\
\hline & Desvio padrão & 16,36 & 8,01 & 15,71 & \\
\hline & $\mathrm{CV}$ & 47,82 & 41,44 & 53,24 & \\
\hline \multirow[t]{3}{*}{ Creatinina (mg/dL) } & Média & 1,51 & 0,80 & 1,30 & $P^{(2)}=0,0801$ \\
\hline & Desvio padrão & 1,43 & 0,30 & 1,25 & \\
\hline & $\mathrm{CV}$ & 95,11 & 37,91 & 95,96 & \\
\hline \multirow[t]{3}{*}{ Sódio (mmol/L) } & Média & 138,71 & 142,33 & 139,80 & $P^{(1)}=0,0171^{*}$ \\
\hline & Desvio padrão & 3,12 & 1,86 & 3,23 & \\
\hline & $\mathrm{CV}$ & 2,25 & 1,31 & 2,31 & \\
\hline \multirow[t]{3}{*}{ Potássio (mmol/L) } & Média & 4,31 & 3,92 & 4,19 & $P^{(1)}=0,0056^{*}$ \\
\hline & Desvio padrão & 0,62 & 0,42 & 0,59 & \\
\hline & $\mathrm{CV}$ & 14,47 & 10,64 & 14,02 & \\
\hline \multirow[t]{3}{*}{ Cálcio (mg/dL) } & Média & 5,96 & 8,20 & 6,24 & $* *$ \\
\hline & Desvio padrão & 2,67 & - & 2,59 & \\
\hline & $\mathrm{CV}$ & 44,79 & - & 41,59 & \\
\hline
\end{tabular}

(1) = através do teste $t$ de Student com variâncias iguais

$\left({ }^{* *}\right)=$ não pode ser determinado devido à existência de apenas um paciente num dos grupos

TABELA 6. Manifestações clínicas pelo estado nutricional dos pacientes com DII

\begin{tabular}{|c|c|c|c|c|c|c|}
\hline \multirow{3}{*}{ Variável } & \multicolumn{4}{|c|}{ Estado nutricional } & & \\
\hline & \multicolumn{2}{|c|}{ Desnutrido } & \multicolumn{2}{|c|}{ Normal } & \multicolumn{2}{|c|}{ Grupo total } \\
\hline & $\mathrm{n}$ & $\%$ & $\mathrm{n}$ & $\%$ & & \\
\hline \multicolumn{7}{|l|}{ Sinais e sintomas } \\
\hline Dor abdominal e diarréia sanguinolenta & 6 & 35,3 & 4 & 57,1 & 10 & 41,7 \\
\hline Vômitos, dor abdominal e fezes com sangue & 4 & 23,5 & 1 & 14,3 & 5 & 20,8 \\
\hline Dor abdominal, astenia e diarréia & 3 & 17,6 & - & - & 3 & 12,5 \\
\hline Esofagite, dor abdominal e fezes com sangue & 2 & 11,8 & 1 & 14,3 & 3 & 12,5 \\
\hline Estenose e aumento do volume abdominal & 1 & 5,9 & - & - & 1 & 4,2 \\
\hline Dor abdominal, desidratação e diarréia & 1 & 5,9 & - & - & 1 & 4,2 \\
\hline Dor abdominal e icterícia & - & - & 1 & 14,3 & 1 & 4,2 \\
\hline Total & 17 & 100,0 & 7 & 100,0 & 24 & 100,0 \\
\hline \multicolumn{7}{|l|}{ Manifestações intra e extra-intestinais } \\
\hline Osteoporose & 4 & 40,0 & - & - & 4 & 26,7 \\
\hline Osteoporose, ileostomia & 1 & 10,0 & - & - & 1 & 6,7 \\
\hline Colostomia & 1 & 10,0 & 1 & 20,0 & 2 & 13,3 \\
\hline Gastrite, esteatose & 1 & 10,0 & - & - & 1 & 6,7 \\
\hline Ileostomia e fístulas & 1 & 10,0 & 1 & 20,0 & 2 & 13,3 \\
\hline Fístula perianal & 1 & 10,0 & - & - & 1 & 6,7 \\
\hline Osteoporose, colostomia & 1 & 10,0 & - & - & 1 & 6,7 \\
\hline Asma, depressão & - & - & 1 & 20,0 & 1 & 6,7 \\
\hline CEP*, úlcera ocular & - & - & 1 & & 1 & 6,7 \\
\hline CEP*, colostomia & - & - & 1 & 20,0 & 1 & 6,7 \\
\hline Total & 10 & 100,0 & 5 & 100,0 & 15 & 100,0 \\
\hline
\end{tabular}

CEP * $=$ colangite esclerosante primária

$\mathrm{CV}=$ coeficiente de variação 
sexo feminino, na faixa etária de 20-50 anos. Dados da literatura indicam que as DII acometem igualmente os sexos, começam a se manifestar com maior frequêencia após os 10 anos de idade e apresentam distribuição bimodal por faixa etária, atingindo picos entre 15 e 25 anos e 55 e 60 anos idade ${ }^{(3,38)}$.

Nesta casuística, grande parte dos pacientes apresentou renda familiar inferior a dois salários mínimos, sendo incompatível com a manutenção de um estado nutricional adequado. Por outro lado, vale ressaltar que a maioria possuía casa própria, residia com seus familiares e tinha o $2^{\circ}$ grau completo, o que talvez possa amenizar os efeitos adversos da baixa renda. Considerando alguns fatores que podem acarretar alterações em certos domínios, principalmente no âmbito social, psicológico e socioprofissional, a proximidade da família associada ao retorno mais rápido ao estilo de vida normal, após períodos de agudização da doença, têm resultados positivos na recuperação dos pacientes ${ }^{(21)}$.

Dados de SOUZA et al. ${ }^{(47)}$ indicam que a determinação da incidência e da prevalência das DII no Brasil é escassa, em função das deficiências no sistema de registro de dados, da falta de informações precisas de casos novos da doença, além da carência de registros da própria população de referência ${ }^{(30)}$. GABURRI et al. ${ }^{(25)}$ e SOUZA et al. ${ }^{(47)}$ forneceram informações representativas da ocorrência dessas enfermidades na região sudeste nas últimas décadas, onde a DC passou a ser mais freqüente que a RCUI. No presente estudo, apesar de limitações ainda maiores na região nordeste, foi observado comportamento diferente, com maior prevalência de casos de RCUI (62,5\%) em relação à DC (37,5\%), o que confirma os resultados apresentados por VIEIRA et al. ${ }^{(51)}$, em que $81 \%$ dos pacientes tratados em ambulatório tinham RCUI, porém com menor gravidade que os casos de DC.

Em relação às características clínicas e localização anatômica das lesões inflamatórias, resultados desta pesquisa concordavam com outros autores ${ }^{(35,50)}$, que similarmente detectaram como principal causa de internamento a diarréia mucosanguinolenta associada à dor abdominal e cólon distal e reto, foram os segmentos intestinais mais afetados, ambos comuns na RCUI. Nos pacientes acometidos com DC foram mais evidentes as complicações intestinais, semelhantes aos achados na literatura, como colostomia, ileostomia e fístulas ${ }^{(18,35,48)}$.

Quanto às manifestações extra-intestinais, é consenso entre pesquisadores ${ }^{(18,40,49)}$ que as afecções mais comuns são osteoarticulares, cutâneas e alterações hepáticas; no entanto, nos últimos anos, existe um interesse crescente pela osteoporose e osteopenia, que podem afetar cerca de $7 \%-42 \%$ dos pacientes com DII, causando importante aumento da morbidade ${ }^{(39)}$. Estes resultados são variáveis entre os estudos publicados, provavelmente devido à heterogeneidade nas populações, duração da enfermidade, tipo de tratamento administrado, critérios diagnósticos utilizados e diferenças metodológicas ${ }^{(34)}$. Neste estudo, foi encontrada prevalência significativa de osteoporose, possivelmente pelo tratamento prolongado à base de corticóides, o que provoca aumento da reabsorção óssea ${ }^{(36)}$ e deficiências nutricionais, associada nesta amostra à condição de inatividade física. Além disso, a resposta inflamatória própria das enfermidades pode favorecer o desenvolvimento desta complicação ${ }^{(28)}$.

No que se refere à dificuldade de diagnóstico das DII, observou-se tempo de diagnóstico superior a 5 anos, como relatado por MISZPUTEN ${ }^{(40)}$, que afirma ser o mesmo muitas vezes confundido com outras afecções intestinais, especialmente durante manifestações iniciais, sendo os casos mais complicados e ricamente sintomáticos, facilmente suspeitos e investigados.

Os pacientes com DII requerem atenção especial quanto ao aspecto nutricional desde o diagnóstico da doença. Neste contexto, a avaliação nutricional permite o reconhecimento precoce da desnutrição e a triagem dos pacientes em risco, o que levará à instituição da terapêutica nutricional adequada. Para tanto, dados obtidos por inquérito dietético, exame físico, mensurações antropométricas e exames bioquímicos devem ser utilizados conjuntamente para um diagnóstico nutricional preciso ${ }^{(53)}$. Entretanto, reconhecem-se muitas dificuldades no estádio destas deficiências em doenças crônicas, sendo difícil discriminar alterações destes parâmetros induzidas pela doença ou pela desnutrição pregressa do paciente ${ }^{(1)}$.

Na população brasileira, dados de inquéritos realizados entre 1975, 1989 e 1996 indicam declínio expressivo da desnutrição em todo país, principalmente na prevalência de adultos com baixo peso, com déficit ponderal em torno de $6 \%$ e na região nordeste $6,8 \%{ }^{(4,41)}$. Segundo CAMPOS et al. ${ }^{(15)}$, a prevalência de desnutrição no grupo de pacientes com DII é alta, variando de $23 \%$ em pacientes ambulatoriais a $85 \%$ em hospitalizados, por exacerbações do quadro clínico. Nesta casuística, o maior percentual de pacientes estava desnutrido pelos dois indicadores. A correlação do estado nutricional, segundo PCT e $\mathrm{CB}$, com sexo, identificou diferenças significativas, com perda da massa gorda e magra mais evidente nas mulheres. Estudo realizado por BURNHAM et al. ${ }^{(1)}$, que associou DC com massa corporal (magra x gorda) em crianças e adultos jovens, evidenciou déficit significante na massa magra, enquanto foi preservada a massa gorda, sendo consistente com o quadro clínico inicial de caquexia.

A associação entre desnutrição e características clínicas revelou percentual elevado de desnutridos com sinais e sintomas mais pronunciados e manifestações extra e intra-intestinais mais freqüentes. Os achados desta série confirmam as descrições publicadas anteriormente por ROLIM ${ }^{(43)}$ e CABRÉ et al. ${ }^{(13)}$, em que encontraram estado nutricional diretamente relacionado com a gravidade com que se manifestam as DII, acarretando prognóstico negativo e deterioração da competência imune. A própria desnutrição agrava a diarréia e/ou má absorção, em função da diminuição da secreção de enzimas pancreáticas e da atividade enzimática na borda em escova das células da mucosa intestinal ${ }^{(42)}$.

Deficiências importantes nestes pacientes estão associadas freqüentemente a conseqüências clínicas adversas, como balanço nitrogenado negativo, limitação da cicatrização de feridas e fístulas, além de descalcificação óssea ${ }^{(15)}$, como relatado por HAN et al. ${ }^{(29)}$, que evidenciaram deficiências nutricionais em pacientes internados e de ambulatórios, com maior freqüência de anemia (54\%-80\%) e hipoalbuminemia (25\%-80\%), observadas no grupo com alto índice de atividade da doença. Semelhante ao encontrado no presente estudo, a hipoalbuminemia pode resultar não só do menor aporte protéico e da anorexia, mas também de reduzida síntese hepática, de perdas intestinais, do catabolismo conseqüente à inflamação, da febre e uso de corticoesteróides 
prolongado $^{(20)}$. Quando se correlacionou a albumina com a desnutrição, observou-se uma tendência ao aumento da albumina nos pacientes desnutridos em todos os graus, resultado semelhante ao referido por CABRAL et al. ${ }^{(12)}$, que não encontraram associação entre as duas características, definindo hipoalbuminemia como indicador pouco sensível de desnutrição, porém específico para detectar doença inflamatória ativa ${ }^{(20,27)}$.

A anemia apresentou alta prevalência em ambos os sexos, o que corrobora os dados de CABRÉ et al. ${ }^{(13)}$, que relatam prevalência em torno de $85 \%$ explicada pela deficiência de ferro, folato, vitamina B12, inflamação crônica, ressecções intestinais e perdas sangüíneas. Correlacionando anemia e estado nutricional, detectou-se que os níveis de hematócrito e hemoglobina mostraram tendência abaixo dos valores de normalidade nos desnutridos de todos os graus, assim como nos eutróficos, constatando-se que a anemia independe do estado nutricional.

\section{CONCLUSÕES}

Apesar das limitações do estudo, estes dados fornecem informações relevantes tanto da ocorrência dessas doenças na região nordeste do país, quanto sua associação freqüente com importantes deficiências nutricionais. Embora antropometricamente os pacientes apresentaram-se eutróficos, o elevado \%PP associado à baixa renda per capita demonstra que esses pacientes estão em grave risco nutricional e complicações clínicas mais acentuadas.

São necessários mais estudos com amostra representativa para caracterizar o perfil epidemiológico desses pacientes, principalmente em regiões norte-nordeste, incluindo análises bioquímicas de transferrina, pré-albumina e capacidade de fixação do ferro, acompanhados da pesquisa da atividade inflamatória, que é de grande significância nas DII.

Salviano FN, Burgos MGPA, Santos EC. Socioeconomic and nutritional profile of patients with inflammatory bowel disease at a university hospital. Arq Gastroenterol. 2007;44(2):99-106.

ABSTRACT - Background - Inflammatory bowel diseases include chronic and relapsing inflammatory disorders, represented by ulcerative proctocolitis and Crohn's disease, commonly associated with malnutrition. Aim - Characterize the nutritional and socioeconomic profile of patients hospitalized at the Pernambuco "Hospital das Clínicas", Recife, PE, Brazil. Methods - Cross-sectional study carried out at the gastroenterology clinic, which was previously approved by the Ethics Commission for studies involving human beings. The methods included clinical history data, socioeconomic conditions and nutritional assessment. Data were subject to statistical analysis (Student's $t$ test for equal and unequal variables). Results - The sample consisted of 24 male and female patients, with a mean age of $43.83 \pm 16.13$ years, mostly married, coming from Recife, with low income, who lived in their own house with relatives. We found a higher prevalence of ulcerative proctocolitis (62.5\%), with diagnosis time of more than 5 years, symptoms of abdominal pain, bloody-mucous diarrhea with 6-9 evacuations/day, with the distal colon being the most affected part. Osteoporosis was present in $26.7 \%$ of cases. Nutritional status was assessed through the body mass index. The weight loss percentage corresponded to $41.7 \%$ and $70.8 \%$, respectively, classified as malnourished, associated or not with high prevalence levels of anemia, hypoalbuminemia and hypocalcemia. Gender correlation evidenced higher triceps fold and arm circumference values in men. Conclusions - Despite the study limitations, data suggest relevant information about the occurrence of these diseases in the northeast of Brazil, as well as about its frequent association with important nutritional deficiencies.

HEADINGS - Inflammatory bowel diseases. Crohn disease. Proctocolitis. Nutritional assessment. Socioeconomic factors. 


\section{REFERÊNCIAS}

1. Alpers DH. Use of macro and micronutrients for nutrition support in inflammatory bowel disease. In: Bistrian BR, JA Walker-Smith JA, editors. Inflammatory bowel diseases. Basel: Karger; c1999. p.155-70. (Nestlé nutrition workshop series. Clinical \& performance programme, v.2).

2. Altman DG. Practical statistics for medical research. London: Chapman and Hall 1991. $611 \mathrm{p}$.

3. Aranceta J, Rodrigo CP. Epidemiologia nutricional de las enfermedades del aparato digestivo. Rev Bras Nutr Clin. 2002;17(Suppl 1):8-13.

4. Batista FM, Rissin A. Deficiências nutricionais: ações específicas do setor saúde para o seu controle. Rio de Janeiro: Cadernos de Saúde Pública;1993. p.130-5.

5. Baumgart DC, Dignass AU. Current biological therapies for inflammatory bowel disease. Curr Pharm Des. 2004;10:4127-47.

6. Blackburn GL, Bistrion BR, Maini BS, Schlamm HT, Smith MF. Nutritional and metabolic assessment of the hospitalized patient. JPEN J Parent Enteral Nutr. 1977;1:11-22.

7. Blackburn GL, Thornton PA. Nutrition assessment of the hospitalized patients. Med Clin North Am. 1979;63:1103-15.

8. Bradesi S, McRoberts JA, Anton PA, Mayer EA. Inflammatory bowel disease and irritable bowel syndrome: separate or unified? Curr Opin Gastroenterol. 2003;19:336-42.

9. Brasil. Ministério da Saúde, Ministério do Planejamento, Orçamento e Gestão. Pesquisa de orçamentos familiares 2002-2003. Rio de Janeiro; 2004

10. Brito CAA, Filgueira NA, Liberato IRO. Doenças inflamatórias intestinais. In: Filgueira NA, Costa JR, Leitão CCS, Lucena VG, Melo HRL, Brito CAA, editores. Condutas em clínica médica. $3^{\text {a }}$ ed. Rio de Janeiro: Medsi; 2004. p.314-28.

11. Burnham JM, Shults J, Semeao E, Foster BJ, Zemel BS, Stallings VA, Leonard MB Body-composition alterations consistent with cachexia in children and young adults with Crohn disease. Am J Clin Nutr. 2005;82:413-20.

12. Cabral VLR, Carvalho L, Miszputen SJ. Importância da albumina sérica na avaliação nutricional e de atividade inflamatória em pacientes com doença de Crohn. Arq Gastroenterol. 2001;38:104-8.

13. Cabré E, Fernadez-Bañares F, Gassul MA. Nutrición en la enfermedad inflamatória intestinal. Rev Bras Nutr Clin. 2002;17(Supl.1):27-34.

14. Campos ACL, Coelho JCU. Suporte nutricional nas doenças inflamatórias intestinais Rev Bras Nutr Clin. 1994;9:55-62.

15. Campos FG, Waitzberg DL, Habr-Gama. A terapia nutricional nas doenças inflamatórias intestinais. In: Campos ACL, editor. Nutrição em cirurgia. São Paulo: Atheneu; 2001. p.185-211. (Clínica Brasileira de Cirurgia - Colégio Brasileiro de Cirurgiões).

16. Campos FG, Waitzberg DL, Teixeira MG, Mucerino DR, Habr-Gama A, Kiss DR Inflammatory bowel diseases: principles of nutritional therapy. Rev Hosp Clin Fac Med São Paulo. 2002;57:187-98.

17. Campos FG, Waitzberg DL, Teixeira MG, Mucerino DR, Kiss DR, Habr-Gama A Pharmacological nutrition in inflammatory bowel diseases. Nutr Hosp. 2003;18:57-64.

18. Campos FG, Teixeira MG. Doença de Crohn. In: Coelho JCU, editor. Aparelho digestivo: clínica e cirúrgica. $3^{\mathrm{a}}$ ed. São Paulo: Atheneu; 2005. p.772-85.

19. Carvalho EB. Avaliação nutricional: a base da escolha terapêutica. In: Manual de suporte nutricional. Rio de Janeiro: Medsi; 1992. p.21-40.

20. Dieleman LA, Heizer WD. Nutritional issues in inflammatory bowel disease. Gastroenterol Clin N Am. 1998;27:435-51.

21. Duclos B, Planchon F, Jouin H, Chamouard P, Schieber JP, Ubrich-Leuilliot M, Baumann R, Weill JP. Répercussion socio-professionnelles de la maladie de Crohn. Gastroenterol Clin Biol. 1990;14:966-72.

22. Ekbom A, Helmick C, Zack M, Adami Ho. The epidemiology of inflammatory bowel disease: a large, population-based study in Sweden. Gastroenterology. 1991;100:350-8.

23. Fiocchi C. Inflammatory bowel disease pathogenesis: therapeutic implications. Chin J Dig Dis. 2005;6:6-9.

24. Fisher RL. Wasting in chronic gastrointestinal diseases. J Nutr. 1999; 129(1 Suppl):252s-5s

25. Gaburri PD, Chebli JMF, Castro LEVV, Ferreira JOD, Lopes MHM, Ribeiro AMB, Alves RA, Froede EC, Oliveira KS, Gaburri AK, Gaburri D, Meirelles GSP, Souza AFM. Epidemiologia, aspectos clínicos e evolutivos da doença de Crohn: estudo de 60 casos. Arq Gastroenterol. 1998;35:240-6.

26. Gassull MA, Fernandez-Banares F, Cabré E, Esteves-Comas M. Enteral nutrition in inflammatory bowel disease. In: Rombeau JL, Rolandelli RH, editors. Clinical nutrition: enteral and tube feeding. Philadelphia: W.B. Saunders; c1997. p.403-16.

27. Geerling BJ, Stockbrugger RW, Brummer RJM. Nutrition and inflammatory bowel disease: an update. Scand J Gastroenterol. 1999;34 Suppl 230:95-105.

28. Ghosh S, Meister D. Osteopenia in inflammatory bowel disease. Research and Clinical Forums. 2000;22:159-70.

29. Han PD, Burke A, Baldassano RN, Rombeau JL, Lichtenstein GR. Nutrition and inflammatory bowel disease. Gastroenterol Clin. 1999;28:423-36.
30. Irvine E, Farrokhyar F, Swarbrick ET. A critical review of epidemiological studies in inflammatory bowel disease. Scand J Gastroenterol. 2001;36:2-16.

31. Jorquera-Plaza F, Paz R, Culebras-Fernandes J. Nutrición en la enfermedad inflamatória intestinal. Rev Bras Nutr Clin. 1998;3:189-200.

32. Kamimura MA, Baxmann A, Sampaio LR, Cuppari L. Avaliação nutricional. In: Cuppari L, editor. Guias de medicina ambulatorial e hospitalar UNIFESP/ Escola Paulista de Medicina - Nutrição: nutrição clínica no adulto. São Paulo: Manole; 2002. p. $71-109$.

33. Kanauchi O, Matsumoto Y, Matsumura M, Fukuoka M, Bamba T. The beneficial effects of microflora, especially obligate anaerobes, and their products on the colonic environment in inflammatory bowel disease. Curr Pharm Des. 2005;8:53-1047.

34. Klaus J, Armbrecht G, Steinkamp M, Bruckel J, Rieber A, Adler G, Reinshagen M, Felsenberg D, Von Tirpitz C. High prevalence of osteoporotic vertebral fractures in patients with Crohn's disease. Gut. 2002;51:654-8.

35. Kronbluth A, Sachar DK, Salomon P. Crohn's disease. In: Feldman M, Scharschimdt $\mathrm{BF}$, Sleisenger MH, editors. Sleisenger \& Fordtran's gastrointestinal and liver disease - pathophysiology, diagnosis and management. 6th ed. Philadelphia: W. B. Saunders, 1998. p.1708-34.

36. Lane NE, Lukert B. The science and therapy of glucocorticoid-induced bone loss. Endocrinol Metab Clin North Am. 1998;27:466-83.

37. Lohman TG, Roche AF, Martorell R, editors. Anthropometric standardization reference manual. Abridged edition. Champaign, IL: Human Kinetics Books; 1991. p.90.

38. Mayberry JF, Rhodes J. Epidemiological aspects of Crohn's disease. Gut. 1984;25:886-99.

39. Menchén L, Ripoll C, Bretón I, Moreno C, Cuerda C, Camblor M, García-Peris P, González-Lara V, Cos E. Osteoporosis y enfermedad inflamatoria intestinal. Nutr Hosp. 2005;20:26-36.

40. Miszputen SJ. Doenças inflamatórias intestinais. In: Miszputen SJ, Schor N, editores Guias de medicina ambulatorial e hospitalar - Gastroenterologia. São Paulo: Manole; 2002. v.1, p.217-31.

41. Monteiro CA. Panorama da nutrição infantil nos anos 90. São Paulo: UNICEF 1996.

42. Pinto PE, Habr-Gama A, Teixeira MG, Ferrini MT, Rodrigues JJG. Moléstia inflamatória intestinal. In: Waitzberg DL, editor. Nutrição oral, enteral e parenteral na prática clínica. $3^{a}$ edição. São Paulo: Atheneu; 2001. p.1361-80.

43. Rolim EG. Nutrição parenteral nas doenças inflamatórias crônicas dos intestinos. Rev Bras Nutr Clin. 1996;11:16-20.

44. Rook GA, Brunet LR. Microbes, immunoregulation, and the gut. Gut. 2005;54:317-20.

45. Shanahan F. Physiological basis for novel drug therapies used to treat the inflammatory bowel diseases I. Pathophysiological basis and prospects for probiotic therapy in inflammatory bowel disease. Am J Physiol Gastrointest Liver Physiol. 2005;288: g417-g21.

46. Siegmund B, Zeitz M. Therapeutic approaches in inflammatory bowel disease based on the immunopathogenesis. Rocz Akad Med Bialymst 2004;49:22-30.

47. Souza MHLP, Troncon LEA, Rodrigues CM, Viana CFG, Onofre PHC, Monteiro RA, Passos, ADF, Martinelli ALC, Meneghelli UG. Evolução da ocorrência (1980-1999) da doença de Crohn e da retocolite ulcerativa idiopática e análise das suas características clínicas em um hospital universitário do sudeste do Brasil. Arq Gastroenterol. 2002;39:98-105.

48. Steinwurz F. Epidemiologia, aspectos clínicos e evolutivos da doença de Crohn. Arq Gastroenterol. 1998;35:237-9.

49. Teixeira-Neto F, Gomes CHR. Doença inflamatória intestinal. In: Teixeira-Neto F, editor. Nutrição clínica. Rio de Janeiro: Guanabara Koogan; 2004. p.360-71.

50. Triantafillidis JK, Emmanouilidis A, Manousos ON, Nicolakis D, Kovevinas M. Clinical patterns of Crohn's disease in Greece: a follow-up study of 155 cases. Digestion. 2000;61:121-8

51. Vieira ACF, Salles P, Carvalho MSA, Cabral PC, Paes AVC, Quirino RM. Perfil nutricional dos pacientes com doença inflamatória intestinal acompanhados no ambulatório de gastroenterologia do hospital das clínicas de Recife. Rev Bras Nutr Clin. 1999;14 Suppl 1:55-6.

52. Vucelic B, Korac B, Sentic M, Milicic D, Hadzic H, Juresa V, Bozikov J, Roktivic I, Bulzevac M, Kovacevic I. Epidemiology of Crohn's disease in Zagreb, Yugoslavia: a ten-year prospective study. Int J Epidemol. 1991;20:216-20.

53. Waitzberg DL, Silva MLT. Diagnóstico das alterações nutricionais na doença inflamatória intestinal. In: Habr-Gama A, editor. Doença inflamatória intestinal. São Paulo: Atheneu; 1997. p.81-7.

54. Zar JH. Biostatistical analysis. 4th edition. New Jersey: Prentice-Hall; 1999. 929 p. 